\section{OWE-025 CATHETER RELATED INFECTIONS IN TYPE 2 INTESTINAL FAILURE PATIENTS ADMITTED TO A NATIONAL CENTRE}

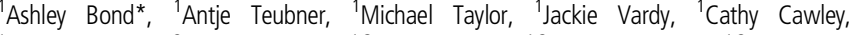
${ }^{1}$ Arun Abraham, ${ }^{2}$ Paul Chadwick, ${ }^{1,3}$ Mattias Soop, ${ }^{1,3}$ Gordon Carlson, ${ }^{1,3}$ Simon Lal. IIntestinal Failure Unit, Salford Royal Foundation Trust, Salford, UK; ${ }^{2}$ Dept of Microbiology, Salford Royal Foundation Trust, Salford, UK; ${ }^{3}$ Manchester Academic Health Sciences Centre, University of Manchester, Manchester, UK
\end{abstract}

\subsection{6/gutjnl-2018-BSGAbstracts.321}

Introduction Management of intestinal failure (IF) requires safe and sustained delivery of parenteral nutrition (PN). Thus, long term safe central venous catheter (CVC) access is vital, with meticulous catheter care and salvage of infected catheters being of prime importance since catheter related blood stream infection (CRBSI) and loss of intravenous access are leading causes of morbidity and mortality in chronic IF. There are, however, no published data on the occurrence and outcomes of CRBSIs in patients admitted with acute severe (type 2) IF.

Methods This is a retrospective observational study conducted between Jan 2011 and July 2017. All new patients with type 2 IF admitted to a national IF Unit during these dates were included. A prospectively maintained database was used to record all confirmed CRBSI cases and clinical data. All new patients admitted with a CVC had paired central and peripheral cultures taken to identify CRBSI. Diagnosis of CRBSI was based on quantitative and qualitative analysis of paired central and peripheral blood cultures. The CVC was used only when CRBSI had been excluded or treated. A standardised 10 to 14 day catheter salvage treatment protocol involving CVC locks and systemic antibiotic administration was used to salvage infected catheters, as appropriate.

Results Of the 509 patients with type 2 IF admitted from another hospital to our IFU during the study period, 341 (54\% female; mean age 54.6 (range 16-86 years) had an indwelling CVC. PICC and tunnelled CVCs were the most common(81.5\%). Surgical complications and mesenteric ischaemia were the most common underlying aetiology. Sixtyfive $(19.1 \%)$ of patients had a diagnosis of CRBSI on the initial screening set of blood cultures and pour plates; the CRBSI had not previously been identified in the referring hospital. A successful CVC salvage rate of 91\% was achieved in this cohort. Over a total of 23548 subsequent catheter days during the 341 patients' stay in the IFU, there was only one CRBSI (0.042 per 1000 catheter days). There was no increased risk of future Home PN related CRBSI $(p=0.09)$ or mortality $(p=0.4)$ in those admitted with a CRBSI at admission, over a follow-up period of 216944 catheter days.

Conclusion All patients should have screening cultures of CVC on admission to an IFU. When CRBSI is present on admission, a high rate of catheter salvage is possible. Stringent CVC care and aseptic strategies in a dedicated IFU can achieve a very low CRBSI rate during the subsequent inpatient stay. CRBSI at index admission to the IFU does not increase risk of future HPN CRBSI or death after discharge

\section{OWE-037 MANAGING ISSUES WITH FOOD-RELATED QUALITY OF LIFE IN INFLAMMATORY BOWEL DISEASE - A QUALITATIVE STUDY}

1,2Wladyslawa Czuber-Dochan*, ${ }^{3}$ Myfanwy Morgan, 'Miranda Lomer, ${ }^{4,5}$ James Lindsay, ${ }^{2}$ Glenn Robert, 'Kevin Whelan. 'King's College London, Faculty of Life Sciences and Medicine, London, UK; ${ }^{2}$ King's College London, Faculty of Nursing, Midwifery and Palliative Care, London, UK; ${ }^{3}$ King's College London, Institute of Pharmacological Sciences, London, UK; ${ }^{4}$ Bart's Health NHS TRust, London, UK; ${ }^{5}$ Queen Mary University of London, London, UK

\subsection{6/gutjnl-2018-BSGAbstracts.322}

Introduction Inflammatory bowel disease (IBD) has a profound impact on diet and nutrition that creates limitations in psychosocial functioning and impacts quality of life (termed foodrelated quality of life, FR-QoL). The issues experienced and the management methods used by patients with IBD and healthcare professionals (HCPs) regarding FR-QoL are not well understood.

Methods Individual semi-structured interviews with 15 IBD patients reporting issues with FR-QoL; and two focus group interviews with $11 \mathrm{HCPs}$ were audio recorded and transcribed verbatim. Pragmatic thematic analysis was used to analyse data, with NVivo 11 used for data management.

Results Fifteen patients with IBD (10 CD/5 UC) were purposively selected from UK hospital outpatient clinics (7 female, mean age $34.4 \mathrm{y}$; range $21-51 \mathrm{y}$ ). Individual interviews ranged from 39-70 min. Eleven HCPs (3 consultant gastroenterologists, 3 IBD registrars, 2 specialist dietitians, 2 IBD specialist nurses and one psychologist) participated in two focus groups over 2 hours each. Patients perceived IBD as having a direct impact on their diet, particularly their food choices and enjoyment of food. This limited their daily life such as going out, socialising with friends and family, or personal relationships. Several factors, including limited understanding of IBD impact on body function and food digestion, fear of triggering a flare through eating, anxiety about making the right food choices, were perceived to contribute to impaired FR-QoL. Patients attempted various methods to improve FR-QoL including trial and error, food avoidance or exclusion, reducing portion size or frequency of eating; but few approaches were perceived to have the desired improvement in FR-QoL. Limited or no dietary advice from HCPs left patients feeling that food-related issues do not receive the same level of attention as medical management. During the focus groups, HCPs identified the factors affecting patients' diet and FR-QoL that needed greater attention and they were: IBD-related (e. g. newly diagnosed, acute inflammation, functional symptoms, strictures and stoma) and non-IBD related (e. g. pregnancy, allergies, likes/dislikes). HCPs acknowledged FR-QoL advice as a low priority in a consultation. HCPs recognised insufficient time in clinical consultations to address more complex issues. Some felt inadequately prepared to offer diet-specific advice, or assumed that other members of the multidisciplinary team provide dietrelated care and advice.

Conclusions Both, patients and HCPs emphasised the need for more individualised care in relation to food and IBD and required quality and timely sources of information. The development and testing of interventions designed to address FRQoL is required. 


\section{OTH-001 GLIADIN PEPTIDE P56-68 ENHANCES EPITHELIAL PERMEABILITY IN A 3D ENTEROID MODEL}

Louise Thompson*, Andra Vaida, Barry Campbell, Carrie Duckworth. University Of Liverpool, Liverpool, UK

\subsection{6/gutjnl-2018-BSGAbstracts.323}

Introduction Increased permeability of the small intestinal epithelium is observed in coeliac disease (CD). ${ }^{1}$ Gluten-derived gliadin peptides are resistant to proteolysis so persist in the gut. They drive $\mathrm{CD}$ pathogenesis by triggering adaptive immunity and via putative direct effects on epithelial cell morphology observed in cell line studies. ${ }^{2}$ Our aim was to ascertain whether gliadin peptides have similar direct effects on 3D enteroids containing all intestinal epithelial cell types, distributed as found in vivo. $\mathrm{C} 57 \mathrm{BL} / 6$ and $\mathrm{BALB} / \mathrm{c}$ mouse strains have different susceptibilities to CD-like pathologies in vivo. ${ }^{3}$ We therefore used enteroids of both strains to examine direct effects of two gliadin peptides on the epithelium in isolation from immune cells.

Methods Enteroid cultures were derived from small intestinal crypts of wild-type $\mathrm{C} 57 \mathrm{BL} / 6$ and $\mathrm{BALB} / \mathrm{c}$ mice according to the method of Sato et al. $^{4}$ Synthetic gliadin peptides P31-43 (LGQQQPFPPQQPY) and P56-68 (LQLQPFPQPQLPY) were purchased from GenScript. For permeability assays, $4 \mathrm{kDa}$ FITC-dextran (FD4) was injected into the enteroid lumen while in Matrigel. After 3.5 hour to stabilise, FD4 fluorescence and bright field images were acquired prior to and hourly post-treatment. The enteroid perimeter was specified from bright field images using ImageJ and the pixel intensity of FD4 fluorescence within this area was measured. For circularity assays, a circularity score was calculated from the enteroid perimeter using ImageJ.

Results $\mathrm{C} 57 \mathrm{BL} / 6$ and $\mathrm{BALB} / \mathrm{c}$ enteroids showed similar epithelial permeability at baseline, observed by loss of FD4 fluorescence over 4 hour $(n=3-5, n=2-5$ organoids). Treatment with $2 \mathrm{mM}$ EGTA significantly increased permeability in all enteroids (all $\mathrm{p} \leq 0.05, \mathrm{n}=3-5, \mathrm{n}=1-6$ enteroids). Gliadin peptide P56-68 $(100 \mu \mathrm{g} / \mathrm{ml})$ enhanced permeability in $\mathrm{C} 57 \mathrm{BL} / 6$ enteroids $(\mathrm{p}<0.05)$, with $\mathrm{BALB} / \mathrm{c}$ enteroids showing the same trend. No effect on permeability was observed in either genotype with P31-43. Despite being leakier, enteroids treated with P56-68 did not exhibit a disrupted morphology. Unlike TNF$\alpha$, which enhanced enteroid circularity in a dose-dependent manner correlating with $\%$ active caspase 3-positive cells, neither peptide altered enteroid circularity over $2 \mathrm{~d}(1-100 \mu \mathrm{g} /$ $\mathrm{ml})$, indicating that they do not induce overt cell death.

Conclusions A peptide fragment of gliadin, P56-68, enhances epithelial permeability in enteroids without inducing cell death. This may contribute to pathophysiology and allow gliadin peptides to access the lamina propria. Further investigation is underway to ascertain the underlying mechanisms.

\section{REFERENCES}

1. Schumann M, et al. Cell Mol Gastroenterol Hepatol 2017;3:150.

2. Clemente MG, et al. Gut 2003;52:218.

3. Papista C, et al. Lab Investig 2012;92:625.

4. Sato T, et al. Nature 2009;459:262.

\section{OTH-002 NUTRIENTS MODULATE CYTOKINE RELEASE FROM EX- VIVO INTESTINAL MUCOSA VIA DISTINCT SENSING PATHWAYS}

Harween Dogra*, Madusha Peiris, Andy Stagg, Ashley Blackshaw, lan R Sanderson. Blizard Institute, Queen Mary University Of London, London, UK

\subsection{6/gutjnl-2018-BSGAbstracts.324}

Introduction Diet may modulate immune responses in health and disease. We hypothesised a role for nutrient sensing pathways. Nutrient sensing enteroendocrine cells (EEC) within intestinal epithelium release local neuroendocrine peptides. ${ }^{1}$ We examined their effects on immune responses in intestinal mucosa.

Methods Three studies were conducted with proximal colon biopsies, comparing whole or de-epithelialised biopsies and dispersed lamina propria to determine contribution of epithelial populations. 1. Expression of genes for nutrient receptors (GPR92, GPR84) and neuroendocrine peptide receptors (GLP1R, GLP2R, NPY1R, NPY2R) was measured using Taqman microfluidic cards $(n=5-7)$. 2. Whole biopsies and deepithelialised lamina propria $(n=12-20)$ incubated with macronutrients protein hydrolysate (whey protein derivative) or lauric acid (medium chain fatty acid), agonists for GPR92 and GPR84 respectively. IL-8, IL-6, TNF $\alpha$, IL-10 and IL-1 $\beta$ profiles were measured in supernatant using a MAGPIX (magnetic bead) multiplex assay. 3. Dispersed lamina propria cells incubated with neuroendocrine peptides GLP1 and NPY $(n=3-5)$ and IL- 8 release measured by ELISA.

Results 1. GPR92 and GPR84 mRNA was detected in both mucosal epithelium and lamina propria. GPR92 expression did not differ but GPR84 expression was significantly greater in epithelium compared with lamina propria $(\mathrm{p}=0.03)$. Expression of neuroendocrine peptide receptors GLP2R, NPY1R and NPY2R was also detected. 2. GPR92 agonist protein hydrolysate significantly reduced IL-8, IL-6 and IL-10 release $\mathrm{p}<0.05$. Removal of epithelium negated this inhibitory effect (figure 1). The GPR84 agonist lauric acid significantly reduced IL-8, IL-6 and IL-10 release within whole biopsies, this inhibitor effect remained after removal of the epithelium, $\mathrm{p}<0.05$ (figure 1). 3. IL-8 release from lamina propria was reduced by $56 \%$ $( \pm 8.5 \%)$ in presence of PY2R agonist NPY compared with medium only (data not shown).
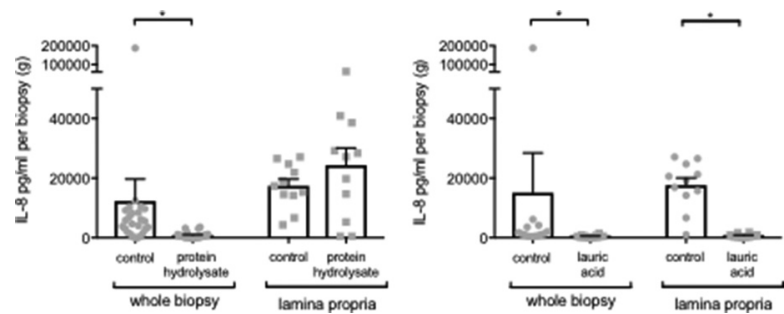

\section{Abstract OTH-002 Figure 1}

Conclusions Nutrient sensing receptors GPR92, GPR84 and neuroendocrine peptide receptors GLP2R, NPY1R and NPY2R are expressed by colonic epithelium and lamina propria. 